\title{
Late Presentations of Cancer in Kashmir, India
}

\author{
Syed Arshad Mustafa ${ }^{1}$, Abdul Waheed Mir ${ }^{2}$, Saquib Zaffar Banday ${ }^{3}$, Malik Tariq Rasool ${ }^{4}$ \\ Received: September 10, 2016 Accepted: December 10, 2016 \\ doi: http://dx.doi.org/10.3329/jemc.v7i1.30752
}

Over $50 \%$ of world cancer burden is born by developing nations. ${ }^{1}$ The proportion of new cancer cases in developing countries is projected to increase from about $56 \%$ of the world total in 2008 to more than $60 \%$ in $2030 .^{2}$ In India it is estimated that over two-thirds of cancer patients report at an advanced stage. ${ }^{3}$ Kashmir valley has a huge cancer burden ${ }^{4}$ with majority of them presenting at a late stage. ${ }^{5}$ Here we present a series of 10 such cases.

\section{Case 1}

Pleomorphic sarcoma on right chest wall extending to right supraclavicular fossa
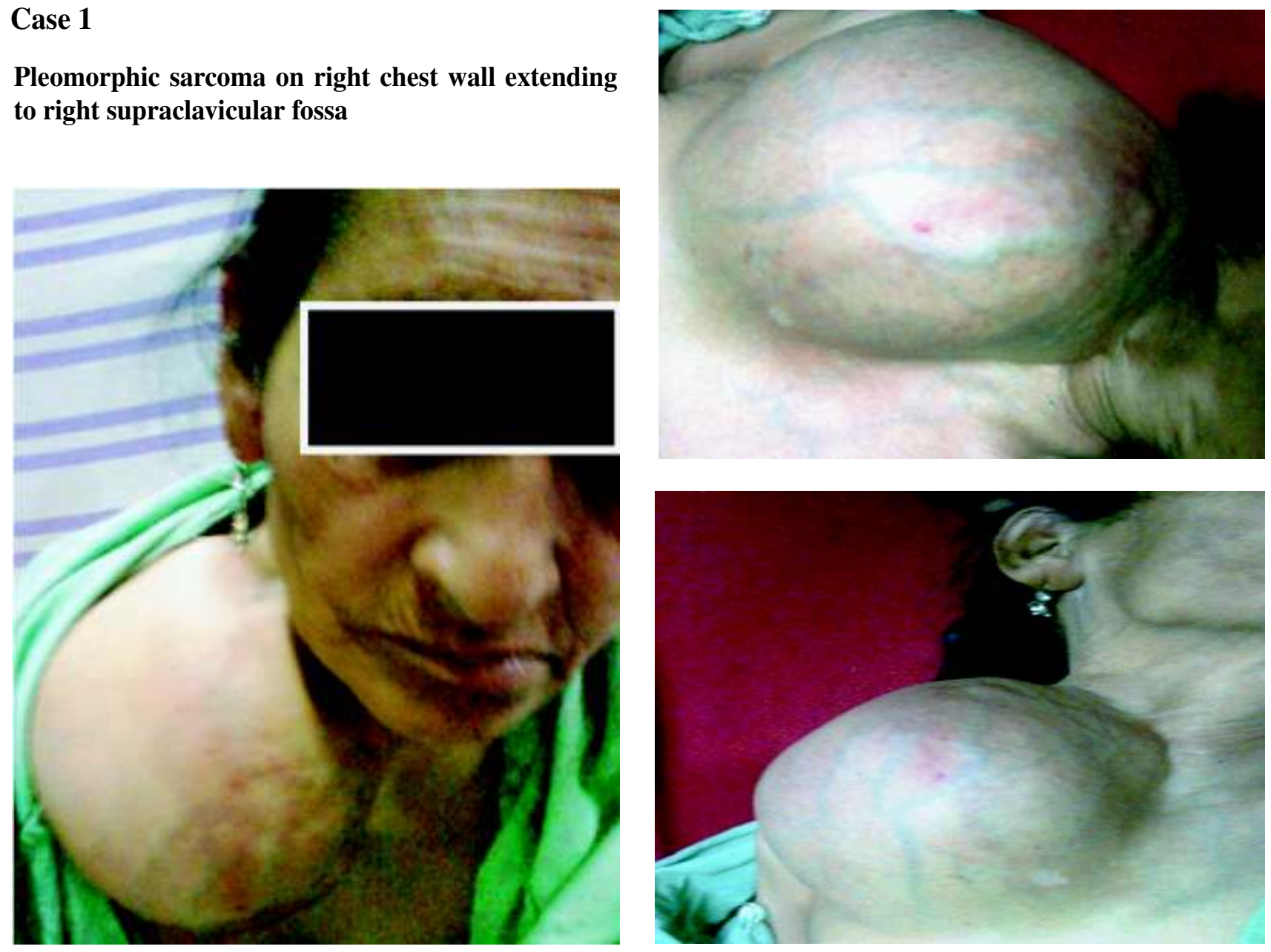

1. Assistant Professor, Department of Radiotherapy, Government Medical College, Srinagar, Jammu \& Kashmir, India

2. Assistant Professor, Department of Surgical Oncology, Sher-E-Kashmir Institute of Medical Sciences Soura, Srinagar, Jaтmu \& Kashmir, India

3. Senior Resident, Department of Medical Oncology, Sher-E- Kashmir Institute of Medical Sciences, Soura, Srinagar, Jammu \& Kashmir, India

4. Assistant Professor, Department of Radiotherapy, Sher-E- Kashmir Institute of Medical Sciences, Soura, Srinagar, Jammu \& Kashmir, India

Correspondence Syed Arshad Mustafa, Email: syedarshad07@gmail.com 
A 50-year-old female presented with an initial slow growing mass over the right infraclavicular area. She had no significant past history. Growth accelerated over a period of two-week time extending to present size of around $8 \times 8 \mathrm{~cm}$ size. Patient ignored the lesion initially and reported only when it interferred in her day to day activities. FNAC was suggestive of malignant round cell tumor. Tru-cut biopsy revealed features of pleomorphic sarcoma. Immunohistochemistry (IHC) was negative for cytokeratin and positive for vimentin. On MRI of neck and chest, lesion was not invading chest or pectoral muscles. Metastatic work-up did not reveal any distant metastasis. Patient was referred to Department of Surgical Oncology for further management.

\section{Case 2}

Advanced nasopharyngeal cancer with metastatic cervical lymph node
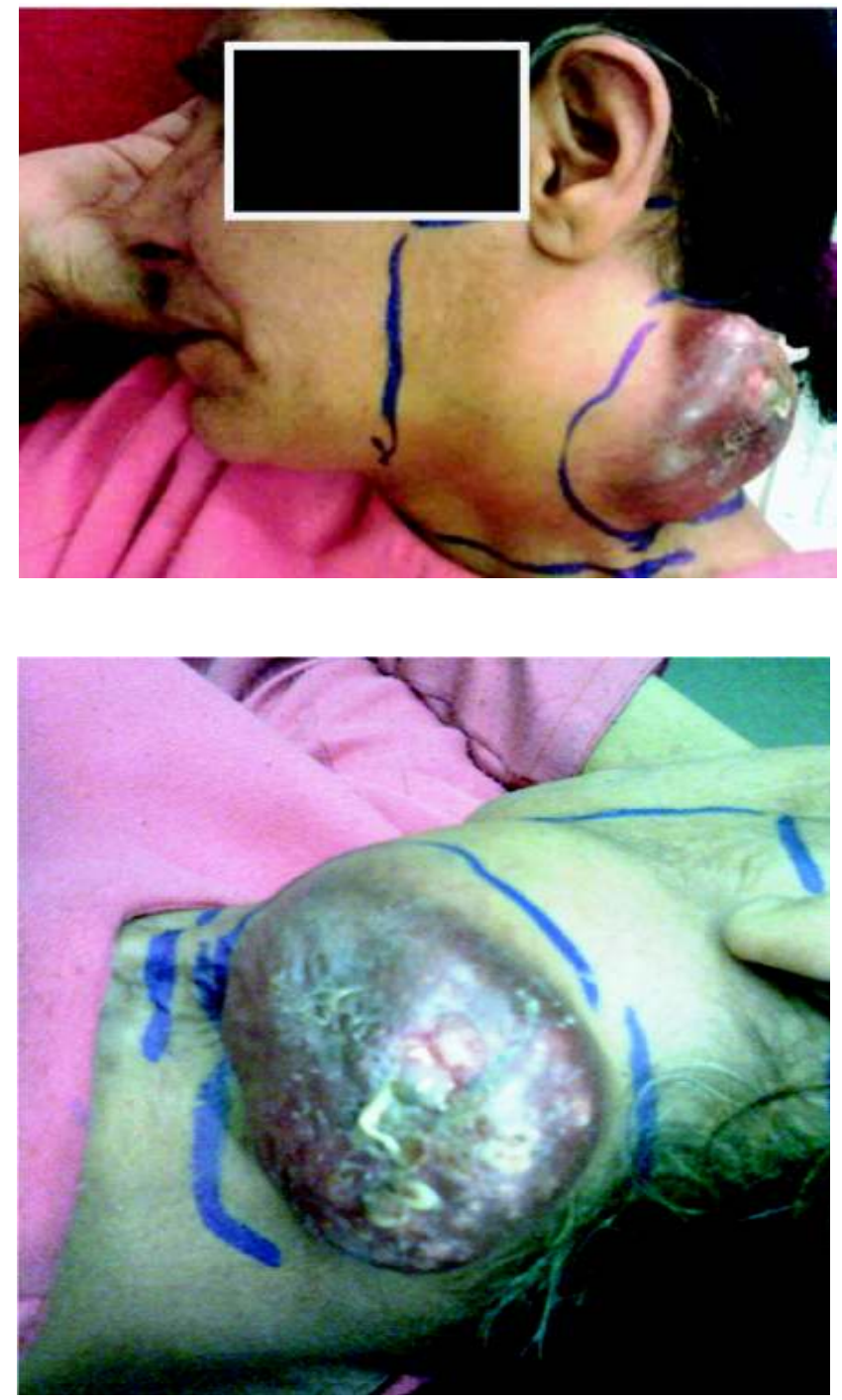

This female patient presented with the history of occasional nasal obstruction and epistaxis. She consulted a doctor in a local hospital where no investigations were advised and she was managed conservatively. She later noticed a swelling over left mid cervical region. FNAC was suggestive of metastatic squamous cell carcinoma with primary in head and neck region. Fibreoptic laryngoscopy revealed fullness in left fossa of Rosenmuller. Patient underwent biopsy of the nasopharyngeal lesion which was suggestive of squamous cell carcinoma (NK type). Patient did not have any distant metastasis on staging work-up. She was planned for concurrent chemo-radiotherapy.

\section{Case 3}

\section{Advanced anaplastic thyroid cancer}

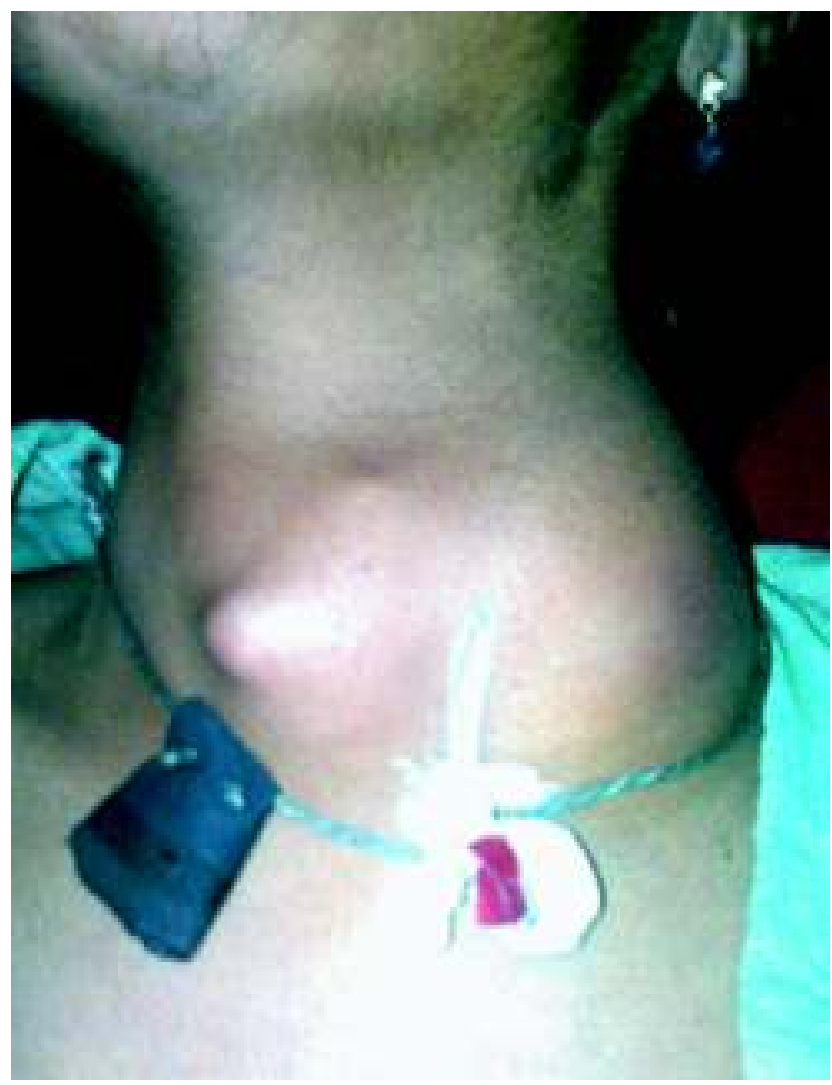

This young female presented with a short (4 weeks) history of progressively increasing mass over left anterior aspect of neck. FNAC was suggestive of anaplastic thyroid cancer. Patient had superior mediastinal extension of the thyroid mass with compression of airway and multiple bilateral pulmonary metastases seen on contrast enhanced CT (CECT) chest. Patient was planned for palliative radiotherapy in view of impending airway compromise. 


\section{Case 4}

Recurrent adenoid cystic carcinoma of parotid gland with skin implants

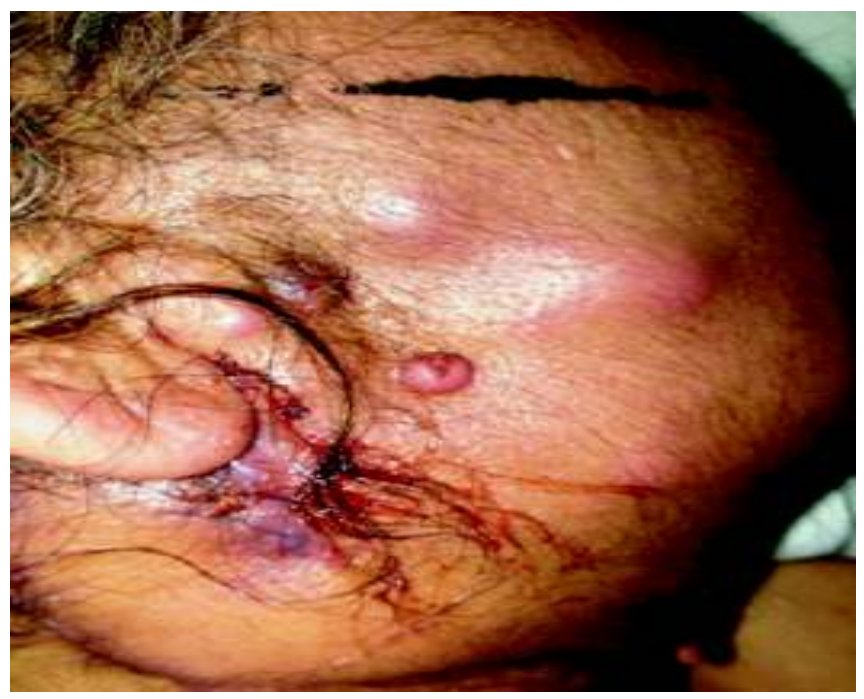

This middle aged female was operated two years back for a swelling over anterior aspect of right ear. Nature of surgery was not known. No biopsy report or blocks was available. She presented to our outpatient department with ear discharge and multiple skin implants over anterior aspect of tragus and previous scar area. She had trismus and otalgia. Cytology from one of the implants revealed features of adenoid cystic carcinoma. Patient was planned for discussion in joint tumor clinic after relevant investigations.

\section{Case 5}

\section{Hodgkin lymphoma with bulky bilateral cervical lymphadenopathy}

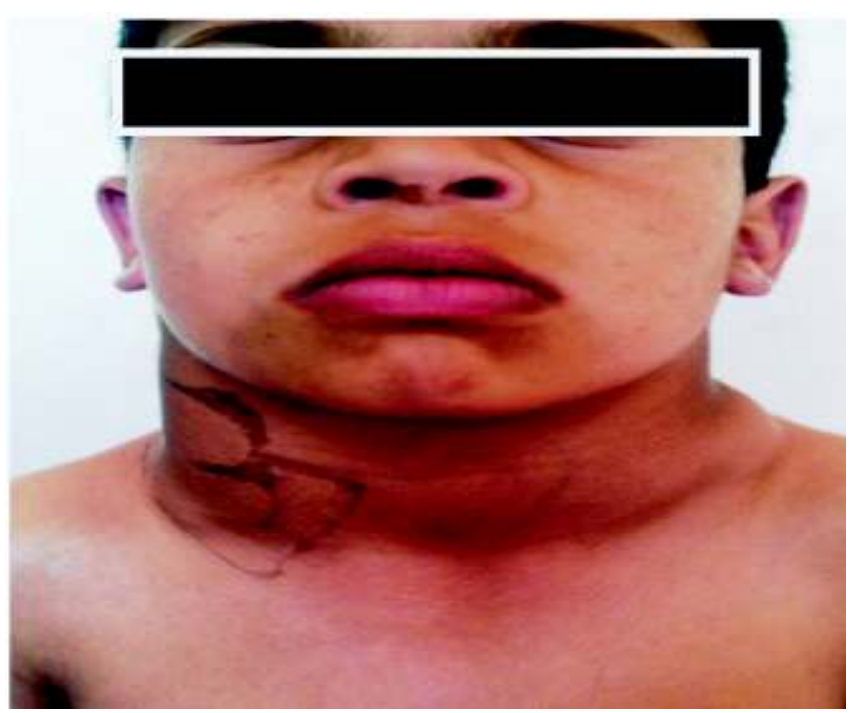

This 14-year-old boy from a remote area in valley presented with history of progressively increasing nodule over right upper neck. Gradually he noticed multiple nodular masses over both aspects of neck. Masses increased in size to present size of $7 x$ and $5 \times 6 \mathrm{~cm}$ over right neck and multiple conglomerate nodes over left side, largest $4 \times 5 \mathrm{~cm}$. Patient had 'B' symptoms of fever and night sweat. CECT neck, chest and abdomen revealed bilateral bulky cervical lymphadenopathy and superior mediastinal nodes. Biopsy from the most accessible neck node reported the features of Hodgkin lymphoma. Patient was planned for chemotherapy.

\section{Case 6}

\section{Advanced breast cancer}

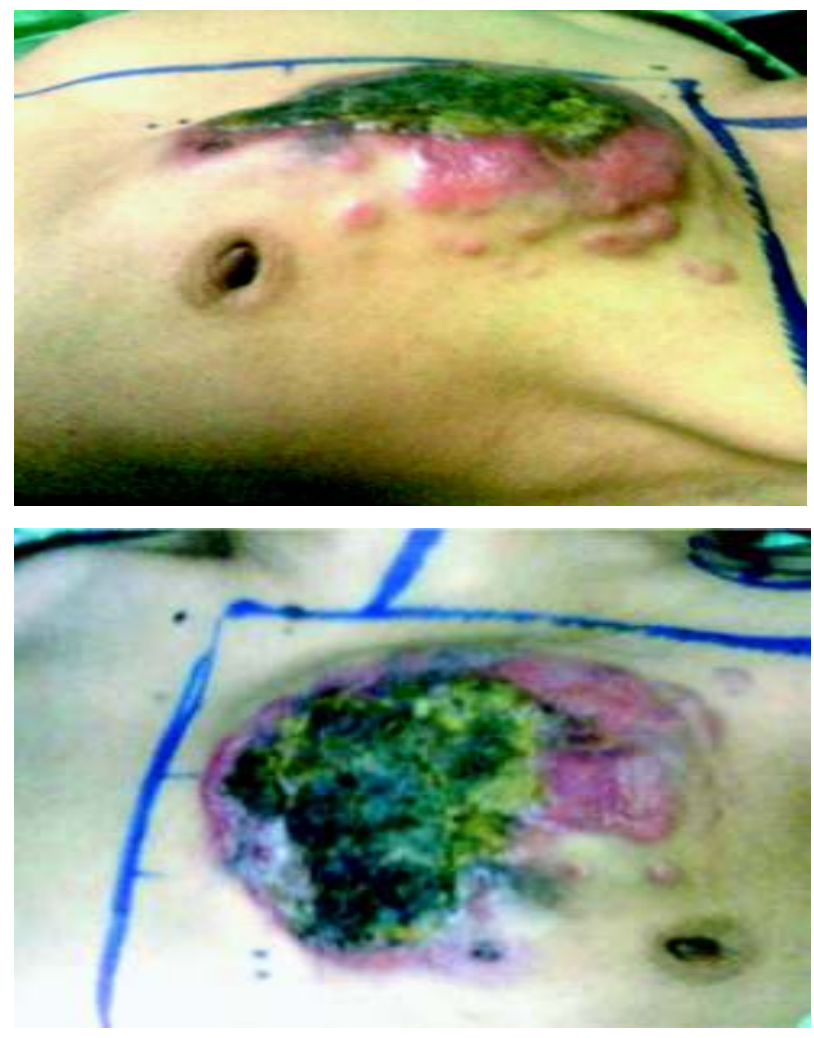

This elderly patient had history of lump in upper inner aspect of left breast for a period of 2.5 years for which she did not consult any physician. She then presented with multiple erythematous skin implants and necrotic area over upper medial aspect of left breast with nipple retraction. Edge biopsy from one of the lesions revealed infiltrating ductal carcinoma. Patient had multiple lung and bone metastases. Immunohistochemistry was negative. She was then planned for palliative radiotherapy in view of low performance score and advanced age. 
Case 7

Cancer oesophagus with tracheo-oesophageal fistula at presentation
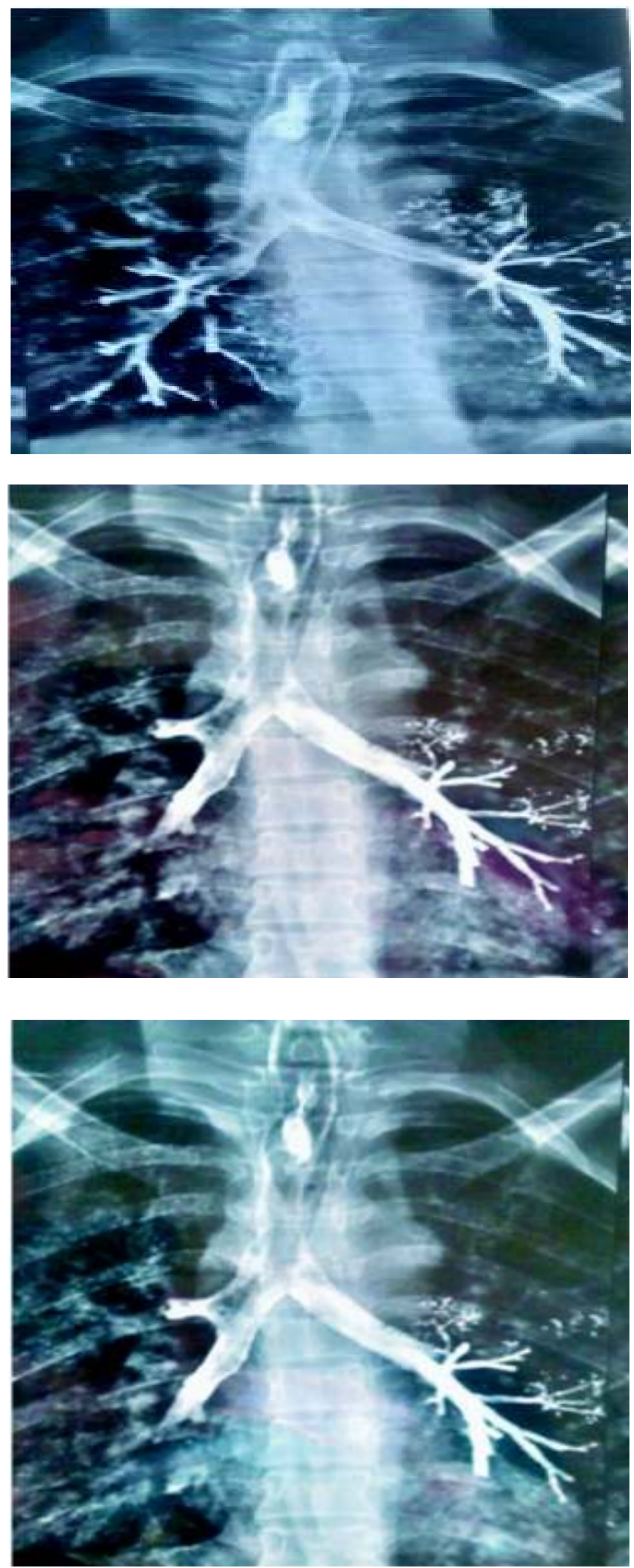

A 65-year-old male, chronic 'hooka'ubble)

smoker, with six months history of progressive dysphagia reported to pulmonologist with history of cough and blood-tinged expectoration. Chest radiography was non-revealing except bilateral reticulopattern shadow. CECT chest revealed proximal dilatation and distal narrowing of middle oesophagus with doubtful communication between oesophagus and trachea suggestive of a fistulous communication. Contrast radiography swallow confirmed the diagnosis of tracheo-oesophageal fistula. Patient was planned for stent insertion as endoscopic biopsy report confirmed it as a case of squamous cell carcinoma of oesophagus.

\section{Case 8}

\section{Advanced breast cancer with multiple skin implants}
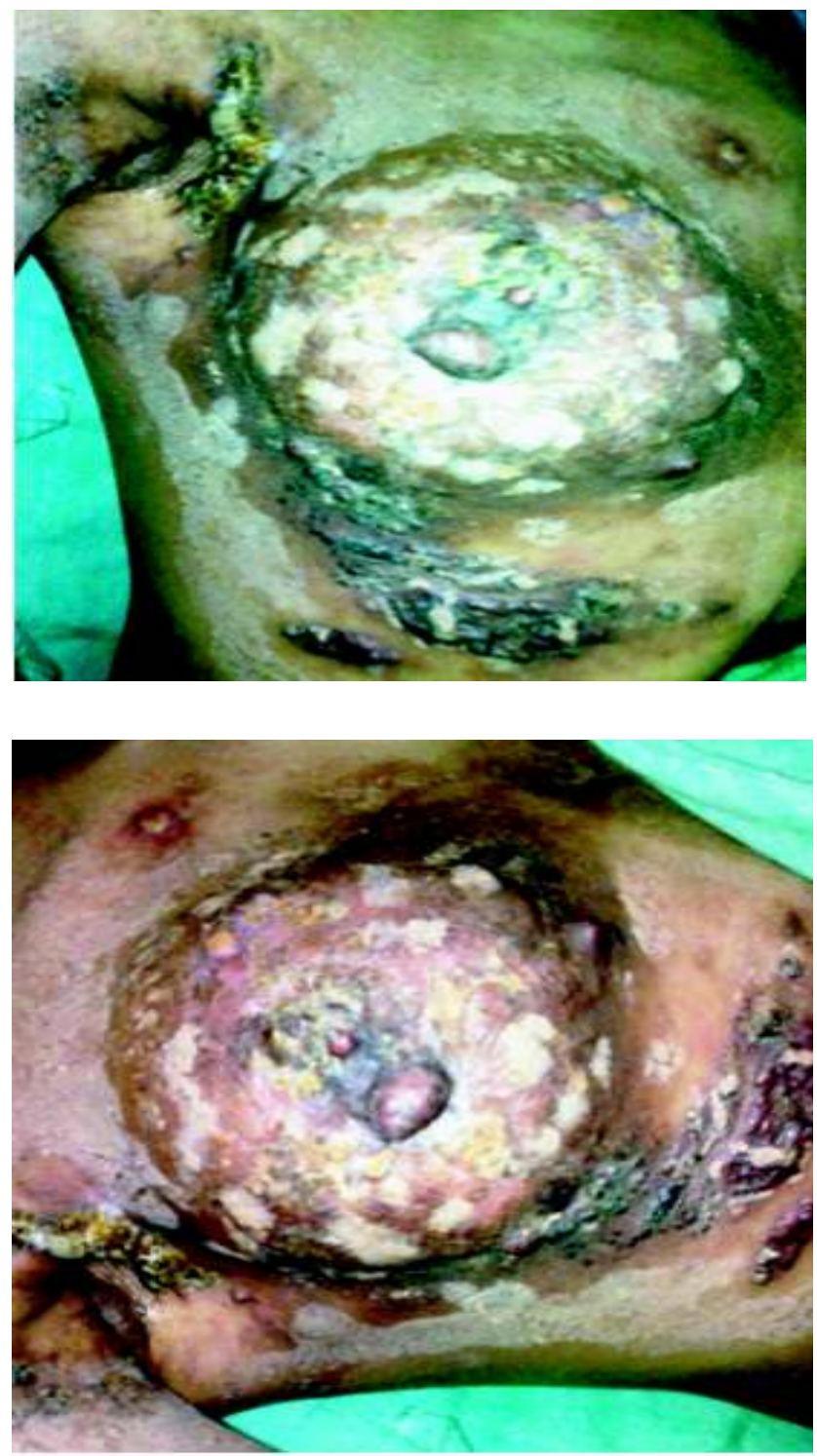
A 42-year-old female presented with history of a painless lump in the retroareolar area of right breast. The lump increased gradually to the size of a walnut with subsequent ulceration of overlying skin. There was history of some operative intervention by a local 'hakeem', over the axillary area (scar with overlying crust appreciable). Later multiple skin ulcerations developed over the entire breast. Patient had clinically palpable right axillary and supraclavicular nodes. Biopsy from one of the breast lesions confirmed the diagnosis of infiltrating ductal carcinoma. There was no evidence of distant visceral metastasis. Patient was planned for toilet mastectomy.

\section{Case 9}

\section{Advanced adamantinoma of mandible}

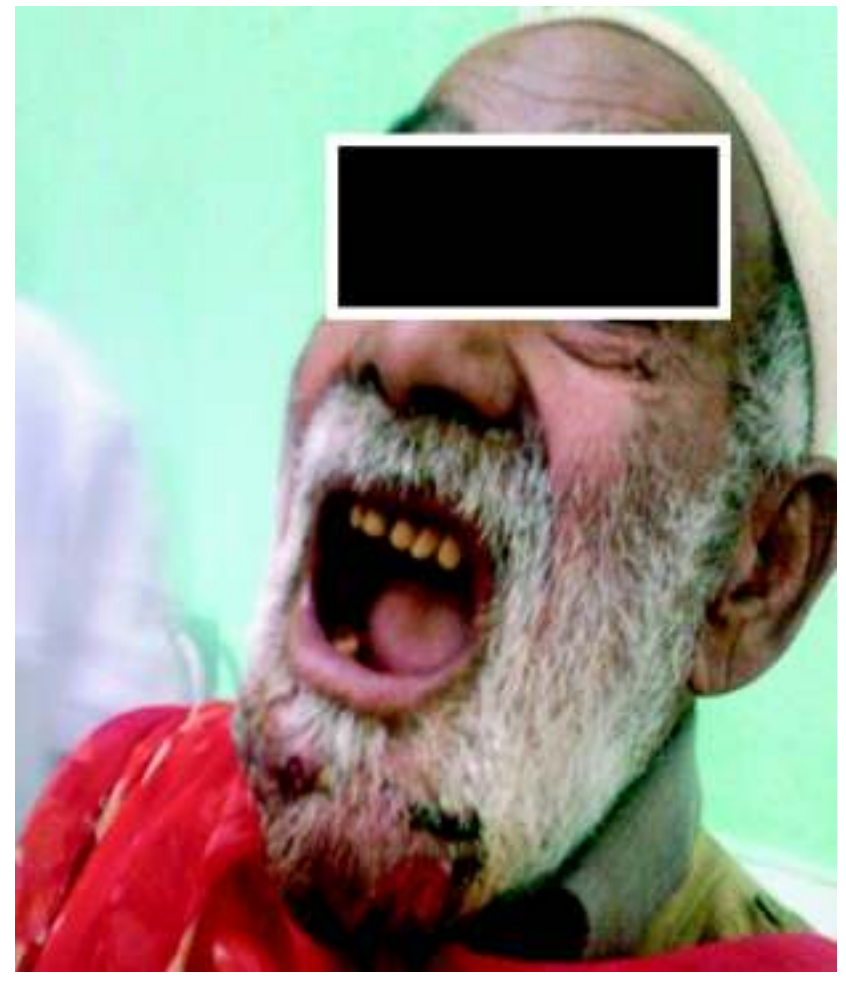

This 70-year-old male presented with pain and swelling over the mandibular area for the last four years. Radiography revealed a mixed sclerotic expansile lesion in mandible with poorly delineated margins. The swelling later increased in size with involvement of overlying skin. Biopsy from the mandibular lesion revealed features suggestive of adamantinoma. Immunohistochemistry was positive for ck 5 and 14 and negative for ck 8 and 18. Patient was planned for radiotherapy as he refused surgery.

\section{Case 10}

\section{Advanced tonsillar lymphoma with right cervical lymphadenopathy}

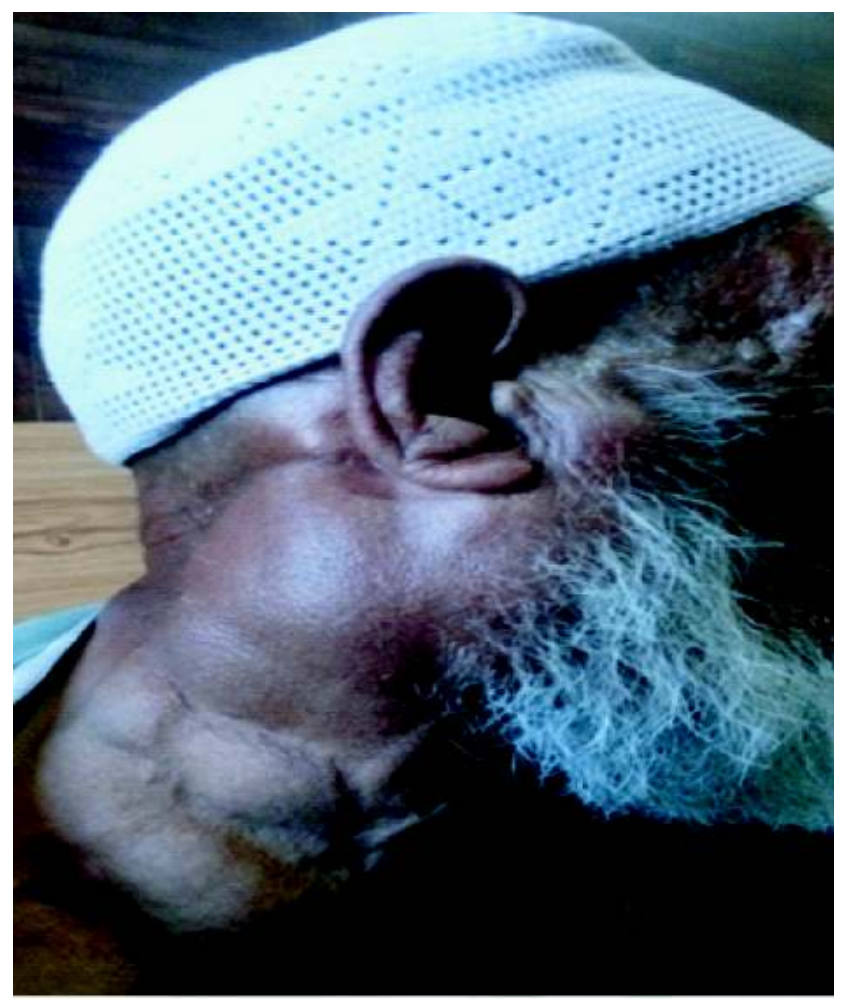

A 62-year-old male non-smoker was evaluated for odynophagia and progressively increasing right sided cervical neck nodes. Oral examination revealed a polypoid mass over the right tonsillar fossa. Biopsy was suggestive of malignant round cell tumor favouring lymphoma. Biopsy from neck node mass was favouring non-Hodgkin lymphoma (DLBCL type). IHC was done favouring DLBCL with CD 20 positivity. CECT done as a staging procedure did not reveal disease anywhere except tonsillar area and cervical node chain. Patient could not afford PET scan. Bone marrow was normal. He was planned for systemic chemo-immunotherapy.

\section{Conclusion}

Various analyses have hinted towards multifactorial causes including patient-related delay, physician-related delay and system or administrative delays for this late presentation of such cases. ${ }^{2}$ The remedy has to be multipronged and multifaceted addressing lacunae at every level as 'The World Health Organisation' states that there are four key components to cancer control which include cancer prevention, early detection, diagnosis, treatment and palliation. 


\section{References}

1. Barton MB, Frommer M, Shafiq J. Role of radiotherapy in cancer control in low-income and middle-income countries. Lancet Oncol 2006; 7: 584-595.

2. Benny PV. Global trends in cancer: developing countries need more focus. International Journal of Preventive and Therapeutic Medicine 2005; 3(2): 32-33.

3. Teli MA, Kuchay SU, Kharadi MY, Lone MM. Late presentation in cancer: who is responsible? JK Practioner 1995; 2(4): $165-168$.

4. R asool MT, Lone MM, Wani ML, Afroz F, Zaffar S, Mohib-ul Haq M. Cancer in Kashmir, India: burden and pattern of disease. J Can Res Ther 2012; 8: 243-246.

5. Mustafa SA, Ismail M, Banday SZ, Patigaroo AR, Malik Tariq Rasool MT, Nabi MG et al. Scenario of palliative radiotherapy in Kashmir. International Journal of Scientific Study 2016; 3(12): 153-159. 